\title{
Self Analysing and Smart Precaution about Lung Diseases
}

K.Kasturi

Ph.D Research Scholar

Vels University

Chennai, India.

kasturi.scs@velsuniv.ac.in
Dr.S.Prasanna

Associate Professor

Vels University

Chennai, India.

prasanna@velsuniv.org

\begin{abstract}
This system using android OS with the help of medical expert system provides advantages to patients, enabling them to access medicinal information and support systems, irrespective of their current location and time. This application is mainly focused for lung diseases and how to get treatment for those particular diseases what we are affected. In this application user once registered can access this application wherever we want. In this if we select diseases preferred medical prescription, precautions, symptoms, and differential diagnosis (Treatment) are available. In this system even location of the specialist's doctors is also available. Once we get details of the doctors after that we can get the location for particular path through GPS from the current location. In this system we can reach the doctor's place and get the treatment as soon as possible.
\end{abstract}

Keywords-Disease diagnosis

\section{INTRODUCTION}

This analysis framework is a specialist framework which is utilized for disentangling the undertaking of specialists. It is a framework that checks a patient at beginning level and proposes the conceivable sicknesses. It begins with enlistment of the patient, clarifies about the lung part and shows all the malady associated with the lung with their side effects, precautionary measures and wanted tablets for the ailment. On the premise of accessible aggregate data, the framework will show the name and the restorative solution of the malady. This framework rearranges assignment of the specialists as well as helps the patients by giving introductory prescriptions to little infections in crisis.

It reminds the possible diseases to the doctor on the basis of symptoms (to overcome human errors like diligence, versatility and tiredness). It can assist a doctor and also help the patient to conduct a diagnosis in order to identify the disease. On finding the disease, a user would be provided with two options.

(1) Medicines, 
(2) Specialist doctor.

Hence in last option it gives user a provision to know about medical centers in neighborhood in case the user is new to the place/town. It is a complete health care management system.

\section{EASE OF USE}

\section{A. Maintanance}

The goals of this help work are to guarantee that the system gets into work untouched with no bug. Game plan must be for environmental changes which may impact the PC or programming system. This is known as the help of the structure. Nowadays there is the fast change in the item world. As a result of this quick change, the structure should be prepared for altering these movements. In our assignment the method can be incorporated without affecting diverse parts of the system. Support expect a key part. The structure will prepared to recognize any change after its use. This structure has been expected to help each new change. Doing this won't impact the system's execution or its precision. This is the last progress in structure life cycle. Here we realize the attempted slip-up free structure into bona fide condition and take off indispensable upgrades, which continues running in an online way. If all else fails, system testing takes, as its input,all of the "organized" programming fragments that have viably passed blend testing and besides the item structure itself consolidated with any material hardware systems. The explanation behind compromise testing is to recognize any anomalies between the item units that are fused together (called social events) or between any of the exhibits and the gear. System testing is a more limited kind of testing; it tries to recognize relinquishes both inside the "amongst social affairs" and moreover inside the structure all things considered.

\section{LITERATURE SURVEY}

In previous research, the study of global refinement has pursued mobile phones via local trajectories. Using these trajectory patterns, the movements of object are forecasted with their location and T-pattern trees [9] are computed. The locations are presented with spatial [8] terms as longitude and latitude values. The Geocode which deliver the values of the location with the help of API along with Google Maps. The related body of work concentrated on Location Based Services [6] for obtaining the location and utilizing it for providing a set of services, where location manager acts as a hook. It offers a wide environment to predict the footprint of the location with proximity alerts. 
Moreover, it affords a clear-cut location with the help of the system termed as GIS. Pioneering work is made towards GIS that furnish the geospatial [1] functionality for many Location Based Services to extract the map information, map visualization and directory based services. There are several studies made towards Location Based Services which are useful to handle Public safety, emergency services, consumer services and enterprise services. The main finding is that, they are probably used in Health care [7] center for emergency services. At first the footprint of the people are made by the mobile sensor which has to be carried by them wherever the moves. It is made by the wireless sensing device which tracks the location of the people by their movement.

These sensors cannot predict the location accurately when the user forgets it and leads to various issues. After that a infrared based spectrum is made in which the footstep of the patient are traced in a home-like environment as well as real home, where the art of localization [12] is made with environmental sensors. This sensing is performed for indoor localization with the application termed as Telehomecare [13], which controls the remote monitoring of patient with pervasive networks.
Now the locations marked for tracking the origin and terminal of the user which is based on path taken by them. The location may arise in any environment where gap between the places must be the shortest path in emergency situation. It can be gained by the Shortest path algorithm [4][5], where the closest facility analysis hand over the route across source and destination for single or multiple landingplace. It can be processed with various algorithms includes Dijkstra's algorithm [4] and Bidirectional ST Algorithm [5].The Dijkstra's algorithm [4] finds the shortest path between the nodes in the graph with different variants. It has a powerful functionality with set of stops where Network Analyst's route solver attempts to find a way at minimum cost. On the other hand, the Bidirectional ST algorithm [5] finds the shortest path from initial to a goal vertex by simultaneously running two searches. The initial vertex is enforced by forward search and the goal vertex is compiled by backward search.

Location are depend on users behavior and the concept NextPlace [10],a location prediction technique which forecast the next location with arrival and residence time based on nonlinear time series analysis. It is a pervasive application that has the capacity to predict the future location of the people and presents 
different location in spatio-temporal [8] point of view. They will extract place via GPS data or Wifi logs. The order-k running average predictor is used to estimate the future values with duration time of last $\mathrm{k}$ visit and interval between $\mathrm{k}$ visits to be averaged. The average value is used to obtain a prediction results of future visits. The WhereNext [9] is similar process that aimed to locate the next location of the moving object with certain accuracy level.

\section{SYSTEM ARCHITECTURE}

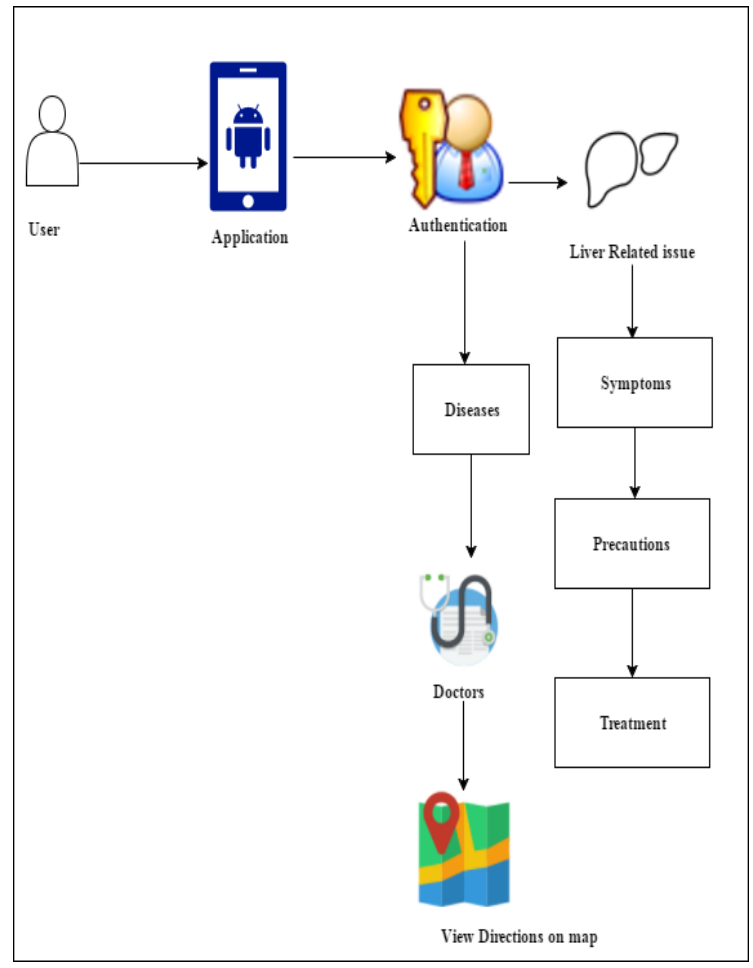

Overall Architecture Diagram

\section{Proposed System}

In the proposed system, providing a platform where a user can login to the application and make appointment with multiple services like hospitals, treatment specialists. The first time user has to sign up with the application and then can access the application with his unique user id and password. The user can select the service and enter the details and purpose of the appointment with the predefined time. This is particularly concentrate for lung affected diseases, Precautions and treatments. Get accurate location for specialist's doctor using GPS.

\section{RESULT}

This application makes the work easier for the patients to detect the disease and also for the doctor. This application not only specifies the specialist doctors of the disease it also helps the users to identify the doctor's destination through the GPS

\section{CONCLUSION}

In this manner, we presume that our Diagnosis System gives answers for the everyday needs and prerequisite in this quick moving and occupied world. It gives stockpiling and recovery offices (In setting with maladies and medicines).This framework guarantees less or no printed material and furthermore gives clients the solace of home. In this framework each data put away and recovered electronically that makes it powerful and gives simple 
recovery of malady data without looking all over. The patient can take utilization of DS sitting at home on their advanced mobile phones and discover drugs at the exceptionally same level.

Online DS is exceptionally valuable for the patients and also specialists in light of the fact that both can get and give data about ailments and drugs from a solitary place on an advanced cell.

\section{VIII.REFERENCES}

[1] Android Programming, and Book release. 002 by Nicolas Gramlich Pg 5 to 60.

[2]Professional Android Application Development by Perth, Western Australia, Reto Meier in London.
[3] Android Open Source Project. Open Handset Alliance:

http://source.android.com/license

[4] Dalvik Debug Bridge Monitor Service: http://developer.android.com/guide/de veloping/tools/ddms.html

[5]Official Android website: http://www.android.com/Open Handset Alliance, website:

http://www.openhandsetalliance.com [6]Java Virtual Machine Tool Interface Spec:http://java.sun.com/javase/6/docs/pla tform/jvmti/jvmti.html

[7]Java Software, Sun Microsystems, Inc. Java Development Kit (JDK). http://java.sun.com/products/jdk/1. 